\title{
Enfoque de competencias. Una alternativa para la profesionalización docente
}

\author{
Meyci de la Cruz Pérez ${ }^{1}$ \\ Marcaolina Pérez Batista ${ }^{2}$ \\ Irina Padrón Delgado ${ }^{3}$
}

\section{RESUMEN}

Los profundos cambios en la realidad mundial contemporánea han condicionado cambios en los sistemas y políticas educacionales de varios países. Es una prioridad que los profesionales que nos desempeñamos en la educación actualicemos nuestros conocimientos y saberes para desarrollar una educación con calidad a la altura de los nuevos tiempos. Es por tanto, para la educación un reto el logro de una alta profesionalización docente. Varios son los modelos de formación del docente como parte de nuestra superación profesional, en este contexto el enfoque de competencias se consolida como una alternativa capaz de tender puente entre lo educativo y lo productivo y es potencializador de nuestro crecimiento personal y social. Es propósito de este artículo argumentar el enfoque de competencia como una alternativa de la profesionalización docente.Palabras clave: Desarrollo, educación, ruralidad, historia, territorio, visión de conjunto.

Palabras claves: Competencias, profesionalización docente.

Recibido: 25 de abril de 2017

Aceptado: 06 de junio de 2017

1 Doctora en Ciencias de la Educación y profesora asistente de la Universidad Máximo Gómez Báez Ciego de Ávila, Cuba. Correo electrónico: meyci@unica.cu

2 Máster en Ciencias de la Educación, en el Instituto Superior Pedagógico "Manuel Ascunce Domenech" de Ciego de Ávila. Profesora Auxiliar, en la Universidad "Máximo Gómez Báez" de Ciego de Ávila.

3 Máster en Ciencias de la Educación, en el Instituto Superior Pedagógico "Manuel Ascunce Domenech" de Ciego de Ávila. Profesora Asistente, en la Universidad "Máximo Gómez Báez" de Ciego de Ávila.

Correo electrónico: irina@unica.cu 


\title{
Focus on Abilities. An alternative to the professionalization
}

\begin{abstract}
The profound changes in world events have shaped contemporary changes in education systems and policies in several countries. It is a priority for education professionals to update our knowledge and skills to develop quality education in line with the new times. Therefore, it is a challenge for education achieving high professionalization. There are various models of teacher training as part of our professional development. In this context the competence approach has established itself as an alternative capable of building a bridge between the educational and productive and it is a propel for our personal and social growth. The intention of this article is to show the competency approach as an alternative for the teacher professionalization.
\end{abstract}

Keywords: Skills, professionalization.

\section{INTRODUCCIÓN}

No es cuestionable para los profesionales de la educación que los profundos cambios en el escenario mundial contemporáneo han condicionado en los finales del siglo XX e inicios del XXI transformaciones en los sistemas y políticas educacionales en varios países.

Al decir de Alem (2006) la educación la vimos por muchos años como una etapa en la vida que se ubicaba en la niñez y en la primera juventud, se impartía en instituciones constituidas a ese efecto (escuelas, colegios, universidades), esto fue válido para un mundo que avanzaba lentamente; donde las revoluciones tecnológicas ocurrían de siglo en siglo, o aún más espaciadamente, pero finalizando el siglo XX todo cambió y cada vez con mayor velocidad, la tecnología que fue válida hace algunos años, hoy ha sido superada en varias generaciones tecnológicas.

En este sentido apunta Yániz y Villardón (2006) que en el mundo actual estamos pasando de una sociedad basada en la industria productiva a otra basada en el conocimiento, atravesando por un estado intermedio que es el de la sociedad de la información.

Bozu y Canto (2009) esclarecen la afirmación anterior cuando señalan que la sociedad del conocimiento no es algo que vivamos actualmente, es un estado final de una etapa evolutiva hacia la que se dirige la sociedad, etapa posterior a la actual era de la información y hacia la que llegaremos por medio de las oportunidades que representan la tecnología de la información y comunicación (TIC) de las sociedades actuales. 
La educación, por tanto, ya no será una etapa en nuestras vidas en la cual lo que se estudia servirá para muchos años de desempeño socio laboral, sino que será ineludible actualizar sistemáticamente las informaciones, conocimientos y saberes.

Cuando egresamos de un centro educacional, nos iniciamos en la práctica profesional y es en ese proceso que validamos la preparación alcanzada y, no pocas veces, descubrimos cuánto nos falta para lograr éxitos mayores en nuestro desempeño; he aquí donde entra la formación permanente que recibimos todos los profesionales como parte de nuestra superación y que nos permitirá perfeccionar el desempeño socio laboral; también nos propiciará estar actualizado en el contexto sociocultural donde nos insertamos profesionalmente, asumiendo novedosos resultados de las investigaciones y las experiencias de avanzada de la práctica empírica, realizada por otros investigadores.

Es por ello que se percibe la necesidad de formarnos para ser capaces de seleccionar, actualizar y utilizar el conocimiento en un contexto específico, que aprendamos en diferentes contextos y modalidades y a lo largo de toda la vida y que podamos entender el potencial de lo que vamos aprendiendo para adaptar el conocimiento a situaciones nuevas (Bricall y Brunner, 2000).

En esta perspectiva que analizamos como la creciente influencia de los resultados de la ciencia en su interacción reciproca con la tecnología y el cambiante ritmo de las exigencias sociales que impactan en el mundo laboral, se acelera la necesidad de que los profesionales docentes que laboramos en la esfera educacional, demandemos de una transformación para asumir nuevos modelos de formación, aprendizaje y socialización orientados a lograr mejores resultados. Por tanto, constituye una labor priorizada en la educación la profesionalización docente a través de la superación profesional.

Asumimos la superación profesional como el impulso a la concepción que tenemos de nosotros mismos y del entorno con las posibilidades que este ofrece y facilita, a la mejora de la autonomía para tomar decisiones frente a los intereses, necesidades y problemas que surgen en la cotidianidad, todo ello para lograr un desarrollo individual y social. El conocimiento que alcanzamos es producto de una búsqueda en común, en donde todos aportamos a partir de nuestros saberes, que es compartido con los saberes de otros profesores través de la búsqueda e investigación participativa.

En este contexto el enfoque de competencia profesional se consolida (CIDEC, 2002) como una alternativa atractiva para impulsar la formación en una dirección que armonice las necesidades de las personas, las empresas y la sociedad en general; dibujando un nuevo paradigma en la relación entre los sistemas educativo y productivo.

Los procesos de formación orientados al desarrollo de competencias emergen para hacer de la educación un servicio más pertinente a las demandas sociales, donde adquiere un significado el ser y saber cómo, sobre el saber y hacer qué, capaz de ofrecer a los profesionales aprendizajes 
socialmente significativos, que los habiliten para operar con eficacia en el contexto específico de las di־ficultades y los retos propios de la época y el país (Clavijo y Fuentes, 2001).

Es para la educación un reto prepararnos para el logro de una alta profesionalización que nos proporcione competencias para la solución de los problemas que emergen de la práctica socio laboral, a través de la superación profesional.

Es nuestra intención en este artículo argumentar el enfoque de competencia como una alternativa para la profesionalización docente, entre tantos existentes en la realidad educativa.

\section{DESARROLLO}

\section{Profesionalización docente: distinciones conceptuales}

El acercamiento, estudio o profundización de cualquier temática se complejiza si se tiene en cuenta lo expresado por Delors (1996) en relación a que el mundo acumula un enorme caudal de conocimientos y tecnologías, que conjuntamente con los que están por descubrir, hacen imposible, que el hombre pueda apropiarse de ellos. Los espacios para que el conocimiento sea socializado crece y el hombre siente cada vez más la necesidad de transmitir experiencias; en tal sentido abordar la revisión del tema propuesto nos resultaron extenso y variado, pues existen tantos discursos como autores han intentado y logrado analizar la problemática.

En la actualidad mucho se habla de los conceptos profesión, profesionales y profesionalización, el discurso realmente se torna complicado cuando se aborda desde las actividades laborales de carácter social.

Según Max Weber (citado por Tenti, 2008), una profesión tiene al menos tres características fundamentales: a) empleo de conocimiento racional técnico; b) autonomía en el ejercicio de la actividad y c) honor estamental, es decir, prestigio y reconocimiento social. En este sentido afirmaba que un profesional vive de y para su profesión, quería decir con ello que el profesional tiene una vocación, es decir, una fuerte orientación afectiva, pero al mismo tiempo, el profesional vive de su profesión, es decir, que de ella obtiene los recursos necesarios para su existencia individual y social.

En esta misma idea argumental Tenti (2008) define lo que sería un profesional, por una parte se caracteriza por la posesión de una serie de conocimientos que por lo general requiere un período de formación más o menos prolongado realizada, de manera general, en una institución especializada; por otra parte, el profesional consagra la mayor parte de su tiempo de trabajo a esa actividad y obtiene de ella los recursos necesarios para su propia reproducción social. Dicho de otro modo, una actividad profesional es una actividad interesada, por eso se dice que el profesional vive del trabajo que realiza. 
En relación al profesional de la educación Goodson y Hargreaves (1996) describen cinco formas de entender la profesionalización docente y que exponemos muy sintéticamente: Profesionalismo clásico (se fundamenta en la comparación de la educación con profesiones de alto prestigio social), Profesionalismo flexible (se sostiene en la cultura de colaboración que surge en las comunidades de prácticos), Profesionalismo práctico (aspira a dar seriedad y prestigio al conocimiento práctico que las personas tienen de la tarea que realizan), Profesionalismo extendido (el profesional deriva sus conocimientos de la relación entre teoría y práctica) y Profesionalismo complejo (los profesionales deberían juzgarse por la complejidad del trabajo que realizan).

Por su parte, Chacón (2004) considera que la profesionalización docente expresa la síntesis entre los valores y cualidades morales que caracterizan la esencia humanista de la labor del maestro o profesor y el dominio de los contenidos de la enseñanza, de los métodos y de las habilidades profesionales que garantizan el desempeño de su función, avalado por la experiencia práctica acumulada, los resultados y los logros obtenidos en su vida profesional.

Entonces la profesionalización docente sería nuestra preparación para la solución de problemas, conflictos y dificultades que de forma más frecuente se presentan durante el ejercicio profesional, el estímulo reflexivo y comprometido con la autopreparación y la colaboración en la construcción colectiva del conocimiento.

Muchos avances educativos relevantes puede aportar sin duda la profesionalización docente, sin embargo debemos estar atentos en situarla como un nuevo mito educativo, así como existen preferencias a la profesionalización, existen elementos que la obstaculizan, el sociólogo argentino Emilio Tenti, en varias de sus obras $(1998,1999,2007,2008)$ detalla cómo estos dificultades han terminado por poner en crisis las viejas identidades de la profesión docente sobre todo en América Latina. Muy apretadamente relacionamos algunas de ellas: la voluntad política de responder a la demanda de educación escolar obligó a expandir sin tomar en cuenta la disponibilidad real de recursos humanos dotados de la formación básica indispensable; la complejidad creciente del trabajo docente y el crecimiento de los conocimientos científicos y tecnológicos necesarios para realizarlo con éxito y la combinación de exclusión social con inclusión escolar, los docentes, se desprofesionalizaron al verse obligados por las circunstancias a asumir nuevas tareas para las cuales no fueron formados convirtiéndose en asistentes sociales entusiastas y no calificados.

\section{Modelos de formación docente para la superación profesional}

Plantea Márquez (2009) que un modelo de formación docente es un diseño para el aprendizaje de la práctica profesional de la enseñanza, que se debe hacer extensivo al profesorado de un mismo nivel educativo pero que debe basarse en la reflexión y la adaptación a los distintos contextos educativos, es decir, debe hacer al docente competente para desenvolverse en los distintos contextos, ámbitos y situaciones educativas. 
A continuación presentamos un listado de modelos de formación docente expuestos por el catedrático Miguel Ángel Zabalza durante una conferencia magistral dictada en la Pontificia Universidad Javeriana de Cali, Colombia en el 2005 que sintetizan la gama de ellos existentes en el ámbito educativo universitario y sobre todo porque constituyen experiencias exitosas aplicadas en diferentes contextos y no una mera clasificación para su estudio.

En primer lugar, hay una serie de modelos basados en el modelo de pares académicos, el cual consiste en que profesores con mucha experiencia asuman la tutoría de profesores noveles que están entrando en el sistema, en España esto se puede observar en algunas universidades como la de Sevilla, la Politécnica de Valencia, la de Santiago de Compostela, los profesores senior van a los cursos de formación con los noveles y hacen las memorias de los mismos, es un trabajo muy interesante por las conexiones de generaciones distintas de profesores.

En segundo lugar hay otros modelos basados en la investigación-acción, que es otro de los sistemas en los cuales los profesores se ponen a elaborar sus materiales o guías para el aprendizaje. Es el estudio sistemático de variables didácticas en los que los profesores podemos estar interesados y que pueden girar alrededor de los errores conceptuales en el campo de las ciencias, las dificultades visuales que puedan tener alumnos de Bellas Artes o el tema de la motivación o aspectos concretos que uno se decida estudiar en su propio contexto.

En tercer lugar proyectos de investigación pedagógica, este es el tema de la Universidad de Alicante, cuestiones que tienen que ver con los procesos didácticos, evaluaciones, analizar resultados de evaluaciones, revisar escritos de los alumnos, hacer un análisis de los ensayos, que vaya mucho más allá del propio análisis como evaluación de la materia que se puede hacer, e incorporación estudiada de nuevas tecnologías, estudiar el impacto de las nuevas tecnologías en el conocimiento que los alumnos van generando.

En cuarto lugar modelos basados en el enriquecimiento doctrinal, esto quiere decir que los profesores vayamos teniendo más conocimiento en cuestiones que tengan que ver con la docencia y en eso están los cursos y talleres, del manejo de bibliografías especializadas. La Universidad Politécnica de Cataluña ha hecho un programa por el cual la universidad regala un libro al profesor que se comprometa a leerlo y comentarlo, para luego colgarlo en la red; muchos profesores están dispuesto a leerse un libro sobre enseñanza basada en problemas, lo comenta y sugiere cosas para la enseñanza, la universidad lo cuelga en la red y el profesor se queda con el libro.

Este enriquecimiento doctrinal se ha ido haciendo con otro sistema que se ha utilizado, que es un modelo de sistema de fichas. Por ejemplo, la Universidad Autónoma de Barcelona puso en marcha un sistema en el cual cada profesor recibía semanalmente una ficha, la ficha era de distintos colores según la temática que trabajaba en ella; habían fichas que podían ser de casos prácticos y entonces en una quincena se analizaba un caso práctico que los profesores recibían; otras fichas podían 
contener artículos aparecidos en la bibliografía internacional, sobre temáticas de interés para el profesorado; había otras en donde se trataban conceptos. Los profesores tenían todo su fichero, en el cual iban recopilando las fichas que quincenalmente se les iba entregando; también habían fichas de tipo conceptual, bibliográfico, de casos prácticos y de propuestas de evaluación o de análisis, éstas las iban metiendo en su sitio de acuerdo al color, unos las tiraban a la papelera y otros muchos las iban coleccionando en la medida que las iban recibiendo.

En quinto lugar modelos basados en la reflexión, por ejemplo, grabarles las clases a los profesores y luego revisarlas con ellos, resulta muy interesante el verse a uno mismo dando su clase y el ir analizando y trabajando esas cosas que en las grabaciones no iba quedando demasiado bien.

En sexto y último lugar modelos basados en la acreditación universitaria, es uno de los modelos que está entrando mucho en Europa a partir de la acreditación y que se entiende como plusvalía personal e institucional. Las universidades, en sus planes estratégicos, van estableciendo el crecimiento académico de su profesorado y entonces la formación aparece ahí determinada por el tipo de condiciones para la acreditación.

Quisimos agregar otro modelo de formación docente con resultados también exitosos en España, Brasil, Estados Unidos: las comunidades de aprendizaje, de las cuales Flecha (1997) plantea que es un modelo abierto, dialógico, participativo y flexible. Las comunidades de aprendizaje se transforman en un espacio de intercambio en el cual cada quien puede aportar al otro parte de lo que necesita, recibir parte de lo que le faltaba y generar juntos provechos compartidos.

\section{Enfoque de competencias como alternativa}

Las competencias como concepto, enfoque y paradigma educativo, emergen en los años ochenta del pasado siglo y se inician como un debate que surge en los países industrializados, sobre la necesidad de mejorar la relación existente entre el sistema educativo y el productivo, sobre todo para educar y capacitar a la mano de obra requerida.

Plantea Díaz (2006) que desde mediados de la década de los noventa, del pasado siglo, en el campo de la educación se pueden encontrar muy diversas formulaciones y expresiones en torno al tema de las competencias, entre ellas destacan: la formación por/de competencias en el estudiantado y el profesorado y propuestas educativas por competencias; de esta manera, la perspectiva centrada en las competencias se presenta como una opción alternativa en el terreno de la educación, que permitirá realizar mejores procesos de formación académica.

De las competencias profesionales se ha hablado a favor y en contra, cierto es que es un concepto de múltiples facetas se ha manejado desde la empresa (la competencia vista en el desempeño eficiente del trabajador); se ha visto como una conformación psicológica compleja (que implica 
componentes motivacionales y afectivos del sujeto) y desde el punto de vista del diseño curricular (formación de un profesional con los conocimientos, procedimientos y actitudes requeridos para ocupar el espacio que le corresponde en la sociedad).

Muchos son los autores que abordan muy acertadamente el tema de las competencias profesionales, extensa sería la lista de ellos desde las diferentes perspectivas antes mencionadas, un análisis integrador y muy ilustrativo resultan los supuestos de Jofré (2009), los cuales sintetizamos seguidamente, él propone los rasgos comunes a los principales componentes de las competencias profesionales, a partir de ellos concluye que las competencias profesionales integran simultáneamente: conocimientos, habilidades y actitudes; que se integran atendiendo a una serie de atributos personales (capacidades, características de la personalidad y recursos individuales); que se manifiestan a nivel de conductas y que poseen una dimensión práctica, de ejecución.

Afirma además, que la utilidad de la competencia profesional radica en que ésta permite evidenciar logros de éxito en los roles ocupacionales; se desarrolla en un contexto determinado, normalmente complejo y cambiante; posee un carácter global para dar respuesta a problemas y situaciones concretos de diversas índole, exige la capacidad de adaptación (flexibilidad) y de trabajo en equipo. Sus principales referentes son social: que van desde lo más genérico hasta el contexto productivo y de la organización del trabajo e individual: relación con los atributos personales que se manifiestan de manera combinada e integrada, considerando también como referencia lo social. Además, la competencia profesional puede adquirirse mediante procesos reflexivos de formación o procesos de aprendizajes en el puesto de trabajo.

Por último, y no menos importante, planteamiento al cual nos unimos porque reafirma la idea que hemos venido argumentado sobre la necesidad de actualización permanente del docente como parte de su superación profesional, que la competencia debe ser evaluada para desarrollar su utilidad, asumiendo que la competencia se platea en un contexto caracterizado por el cambio, ésta evoluciona y por tanto, es necesaria su evaluación, es decir, ser competente aquí y ahora, no significa ser competente en una situación o contexto diverso al que se tiene aquí y ahora.

Nos parece adecuado agregar a esta afirmación, lo expresado por Pavié (2011) porque también constituye sustento de la idea tratada y es que las competencias profesionales no se adquieren en una etapa de formación inicial y se aplican sin más, sino que se crean y se recrean continuamente en la práctica profesional, y además de todo lo anterior, contienen un fuerte componente reflexivo y ético relacionado con la ejecución profesional a la que se vincula.

El enfoque de competencias como alternativa en la formación del profesorado viene teniendo una progresiva importancia, ello está dado en una mayor consideración de nuestro trabajo como una profesión, que un perfil profesional específico y distinto al de otros profesionales. No resulta novedoso, por tanto, decir que nuestro rol profesional está cambiando, permanentemente se abordan las nuevas demandas al perfil profesional del docente. 
La gestión y monitoreo de los distintos tipos de competencias docentes se estructuran por medio de un perfil profesional, el que puede traducirse en términos de competencias de diverso tipo organizadas en dominios de ejercicio profesional y de formación. Estos aspectos son normalmente traducidos en términos de capacidades, tareas y funciones que emplean y desarrollan en el ejercicio de su respectiva profesión. Definido así, el perfil constituye la descripción del conjunto de los atributos de un rol o función profesional en virtud de competencias asociadas a las prácticas de la profesión (Aravena, 2010).

Afirman Zabalza y Zabalza (2012) que no es fácil ser profesor/a en ninguna etapa del sistema educativo debido a le exigencia de combinar tradición y posmodernidad en el ejercicio profesional. Esto implica la necesidad de acomodarse a un nuevo perfil docente. Este nuevo perfil implicará que el docente sea una persona culta, que domine su materia; además, han de ser profesionales técnicos que dominen las competencias básicas de su trabajo como educadores; también, buenos artesanos, capaces de elaborar materiales didácticos propios, de generar recursos, de emplear efectivamente las tecnologías; por supuesto, que sean profesionales reflexivos capaces de documentar y revisar sus prácticas y, finalmente, actores sociales relevantes y comprometidos.

Es por ello que las instituciones educativas deben ofrecer al profesorado posibilidades de formación que conduzcan a construir competencias para identificar y solucionar problemas, conectar diferentes saberes y darles un sentido. Pero para que una política de profesionalización de los docentes sea exitosa no sólo debe basarse en un análisis de los factores objetivos que condicionan nuevos desafíos a la actividad del docente, sino que también debe consultar también sus opiniones, actitudes, valoraciones, expectativas, etc. acerca de aspectos sustantivos de su propia actividad y acerca del contexto en el que la realiza.

\section{CONCLUSIONES}

En el artículo evidenciamos como el proceso de formación por compe $\neg$ tencias busca desarrollar en el individuo capaci $\neg$ dades para hacer frente a toda clase de circunstancias y resol $\neg$ ver problemas con eficacia, eficiencia, efectividad y expectatibilidad en el contexto de su crecimiento personal y social.

El perfil del profesorado viene determinado por el concepto de competencia que es un proceso más flexible que se va alcanzando a lo largo del desarrollo de la profesión, en nuestra opinión nos atrevemos a señalar 10 competencias docentes que debe poseer el profesorado:

1. lanificar el proceso de enseñanza aprendizaje.

2. Atender de forma centrada al estudiante.

3. Motivar y comprometer activamente a los estudiantes con su proceso de aprendizaje y las actividades.

4. Tutorizar. 
5. Tener motivación al logro.

6. Trabajar en equipo.

7. Usar eficientemente las nuevas tecnologías.

8. Potenciar el autoaprendizaje y el desarrollo profesional.

9. Colaborar en la gestión del centro.

10. Desplegar actitudes y valores.

\section{BIBLIOGRAFÍA}

Alem, M. J. (2006). Base de la formación y capacitación en las organizaciones. Recuperado de http://www.monografía.com

Aravena, A. (2010). Competencias profesionales de un docente. Recuperado de http://www. elnaveghable.cl/admin/render/noticia

Bozu, Z. y Canto, P. J. (2009). El profesorado universitario en la sociedad del conocimiento: Competencias profesionales docentes. Revista de formación e innovación educativa universitaria, 2 (2), 87-97.

Bricall, J. M. y Brunner, J. J. (2000). Universidad siglo XXI: Europa y América Latina. Regulación y financiamiento. Documento Columbis sobre gestión universitaria.

CIDEC. (2002). Competencias profesionales. Enfoques y modelos a debate. Cuaderno de trabajo, 27.

Clavijo, G. A. y Fuentes, H. (2001). Diseño curricular y evaluación basados en competencias. Universidad de Oriente, Santiago de Cuba, Cuba.

Chacón, N. (2004). Ética y educación en tiempos de globalización desde la perspectiva cubana. Formación del profesorado en Cuba.

Delors, J. (1996). La educación encierra un tesoro. España: Grupo Santillana de Ediciones.

Díaz Barriga, A. (2006). El enfoque de competencias en educación: ¿Una alternativa o un disfraz de cambio?. Revista perfiles educativos, 28 (111), 7-36.

Flecha, R. (1997). Compartiendo palabras: El aprendizaje de las personas adultas a través el diálogo. Barcelona: Paidós.

Goodson, I. y Hardgreaves, A. (1996).Teachers' professional Lives: Aspirations and Actualities. En I. Goodson y A. Hardgreaves, (Eds.), Teacher's Professional Lives. London: Falmer Press.

Jofré, G. (2009). Competencias profesionales de los docentes de enseñanza media de Chile: Un análisis desde las percepciones de los implicados. (Tesis doctoral). Universidad de Barcelona, España.

Márquez, A. C. (2009). La formación inicial para el nuevo perfil del docente de secundaria: Relación entre la teoría y la práctica. (Tesis doctoral). Málaga, España.

Pavié, A. (2011). Formación docente: Hacia una definición del concepto de competencias profesionales docentes. REIFOR, 4 (1), 67-80.

Tenti, E. (2008). Sociología de la profesionalización docente. (Seminario internacional: Profesionalizar a los profesores sin formación inicial: Puntos de referencia para actuar). 
Villardón, L. y Yániz, C. (2006). Planificar desde competencias para promover el aprendizaje: El reto de la sociedad del conocimiento para el profesorado universitario. Universidad de Deusto, Bilbao, España.

Zabalza, M. A. (2005). Competencias docentes. (Conferencia en la Pontificia Universidad Javeriana de Cali). Colombia.

Zabalza, M. A. y Zabalza, M. A. (2012). Profesoras y profesión docente: Entre el ser y el estar. Madrid: Narcea. 